\title{
Pensar en público... Enseñar a pensar
}

Thinking in Public... Teaching to Think

Penser en publique... Apprendre à penser

Pensar em público... Ensinar a pensar

Fecha de recepción: 10 DE NOVIEMBRE DE 2013/ Fecha de aceptación: 23 DE ENERO DE 2014

Encuentre este artículo en http://magisinvestigacioneducacion.javeriana.edu.co/

doi:10.11144/Javeriana.M6-13.PEEP

Escrito por Fabiola Cabra-Torres Pontificia Universidad JaVeriana Bogotá, Colombia

f.cabra@javeriana.edu.co

\section{Guillermo Hoyos-Vásquez: impronta de un intelectual que pensó en público sobre grandes problemas de las Ciencias Sociales y Humanas}

Esta breve reseña sobre la impronta intelectual del profesor Guillermo Hoyos-Vásquez pretende recoger algunos de los momentos que compartimos con él, estudiantes y profesores del doctorado en Ciencias Sociales y Humanas, de la Universidad Javeriana. Hoy queremos rendirle homenaje por todo aquello que nos legó: su visión crítica de la academia y la lucidez para reflexionar sobre los problemas más profundos y álgidos de Colombia, así como su ejemplar compromiso y esfuerzo constante por enseñar a pensar.

Para muchos de sus estudiantes, discípulos y colegas, el profesor Hoyos-Vásquez representó la figura del "auténtico maestro e intelectual": para recordar las palabras de Heidegger (2010), "Ilegar a ser maestro es una cosa muy elevada, y, desde luego, es muy distinto de llegar a ser un profesor famoso" (p. 78). Además de ser un destacado académico e investigador y un filósofo prominente de nuestro país, en su faceta humana resalta su profunda vocación educadora. Se esmeró de forma genuina para que su erudición filosófica estuviese al servicio del otro, siempre con el fin de despertar la curiosidad, fomentar el examen crítico y el pensamiento deliberativo y cultivar la razón del actuar comunicativo. Nos enseñó que pensar es un acto de responsabilidad pública y ciudadana y que la academia ha de ser un ethos privilegiado para "pensar en público".

Guillermo Hoyos-Vásquez encarnó profundas virtudes intelectuales y morales y recibió múltiples

Para citar este artículo / To cite this article / Pour citer cet article / Para citar este artigo

Cabra-Torres, F. (2014). Pensar en público... Enseñar a pensar. magis, Revista Internacional de Investigación en Educación, 6 (13), $177-184$. 
reconocimientos por su vocación de educador. En los últimos años de su vida, recibió el Premio Nacional de Educación Francisca Radke (2010) y la Condecoración Simón Bolívar, Orden Gran Maestro, en la ceremonia Los mejores en Educación 2012, otorgada por el Ministerio de Educación.

En su trayectoria como director del Instituto de Estudios Sociales y Culturales Pensar, promovió durante casi una década el espacio de discusión denominado Pensar en público, término que, según el profesor HoyosVásquez, se lo debemos al filósofo español Manuel Reyes Mate, uno de los que manifestaron su deseo de pensar en público desde la perspectiva de las víctimas (Hoyos-Vásquez, 2008). Para Reyes Mate, lo que ha dominado en la historia, la política y el Derecho ha sido la invisibilización de las víctimas, de modo que se hace necesario acudir a la memoria -imperativo filosófico - para retornarles su derecho a pedir justicia e iniciar un proceso de reparación y reconciliación.

En los últimos años de vida, como director del Instituto de Bioética y promotor de importantes debates en este campo, advirtió sobre el peligro de reducir la bioética a protocolos, códigos y comités de ética médica o jurídica, frente a lo cual propuso con vehemencia situarla en relación con las raíces de la filosofía moral, de la política y del Derecho, a fin de liberarla para hacerla un saber de ciudadanas y ciudadanos en una sociedad civil en búsqueda de emancipación social, cultural y política (Hoyos-Vásquez, 2012).

En el año 2007, fue llamado a integrar el equipo proponente del doctorado en Ciencias Sociales y Humanas, programa que está en funcionamiento desde 2008. Algunos de sus importantes aportes a la formación doctoral y su trabajo en seminarios y cursos doctorales con el grupo de profesores que conformamos el Eje 3: Sociedad de la información, comunicación y procesos socio-educativos, se traen a la memoria para recordar sus profundas enseñanzas y la huella indeleble que dejó en sus colegas más cercanos.

\section{Las ciencias sociales y el diálogo entre culturas}

Un equipo de profesores de la Pontificia Universidad Javeriana', con el acompañamiento de Guillermo Hoyos-Vásquez, emprendió la tarea de elaborar y sustentar el documento de la propuesta del doctorado en Ciencias Sociales y Humanas. Este programa pretendía, desde los inicios de la discusión en torno a la interdisciplinariedad, retomar una tradición muy propia de la Universidad Javeriana, plasmada en la creación de la Facultad de Estudios Interdisciplinarios (FEI) a principios de la década del setenta, promovida bajo el liderazgo de Alfonso Borrero Cabal, S. J.

Como miembro y gestor del programa, Guillermo Hoyos-Vásquez defendió un sentido de interdisciplinariedad entendida como diálogo entre las Ciencias Sociales, la Filosofía y aquellos campos de saber comprometidos con el cultivo de la humanidad —aludiendo al trabajo de la filósofa estadounidense Martha Nussbaum- En su opinión, con la creación del programa se pretendía transformar la idea fundacional de la investigación y el posgrado interdisciplinar en un doctorado en Ciencias Sociales y Humanas, en donde el sentido de ciencia social fuese comprendido de modo muy amplio y el de humanidades fuese llevado al diálogo con los discursos científicos; en sus palabras se trataba de "un horizonte, una especie de

1 El grupo proponente estaba conformado por: Gloria Marciales (Facultad de Psicología) Rodrigo Lozada (Facultad de Ciencias Políticas y Relaciones Internacionales), Rafael Díaz (Facultad de Ciencias Sociales), Jesús Martín-Barbero y Luis Ignacio Sierra (Facultad de Comunicación y Lenguaje), Fabiola Cabra-Torres (Facultad de Educación), César Ferrari (Facultad de Ciencias Económicas y Administrativas) y Guillermo Hoyos-Vásquez (director del Instituto Pensar). 
utopía intelectual que jalone los diversos saberes en un diálogo comprensivo y articulador de las ciencias, las culturas y los diversos discursos". Así quedó plasmado en el documento fundante del doctorado:

Lo que se busca contra todo nominalismo, con la denominación en Ciencias Sociales y Humanas, es un "programa" en el sentido estricto del término, a nivel de doctorado, en el que se logre el mayor grado de interdisciplinariedad posible para la explicación y la comprensión de aquellos temas, problemas y tareas, de la sociedad y de la cultura, que exigen un discurso desde las diversas ciencias sociales y desde las humanidades, las artes, la filosofía y la teología. Se busca entonces relacionar y conjugar estos saberes de suerte que lo que constituyen las fortalezas de unas y otras como "ciencias" haga posible la complementariedad, animada por su disposición para abordar las problemáticas sociales desde su complejidad y multidimensionalidad.

Sólo a modo de ilustración para justificar el nombre de este programa, cabe retomar el libro de la filósofa norteamericana Martha C. Nussbaum, El cultivo de la humanidad. Una defensa clásica de la reforma de la educación superior, quien después de años de investigación, lecturas, visitas y conversaciones en numerosas y diferentes universidades de su país, propone para reformar la educación superior en el horizonte del "cultivo de la humanidad" tres habilidades: la actitud socrática de una vida examinada, una perspectiva cosmopolita y una imaginación narrativa (Marciales-Vivas, 2008, p. 32).

Desde la creación del programa doctoral se tuvo la convicción y la necesidad de proyectar una idea de inclusión desde las Ciencias Sociales y Humanas que diera lugar a la diversidad de disciplinas, voces, enfoques, lo cual implicaba abrir los diálogos y cuestionar permanentemente la concepción reduccionista y unilateral de la ciencia, la técnica y la tecnología que se instaló con la modernidad y en particular con el discurso hegemónico que decretó la antinomia entre las ciencias de la naturaleza y las ciencias del espíritu.

En su discurso "Manifiesto por las ciencias sociales y las humanidades" (Hoyos-Vásquez, 2011), alude al informe de la Comisión Gulbenkian para la reestructuración de las Ciencias Sociales, Abrir las Ciencias Sociales (1996), coordinado por Immanuel Wallerstein, para señalar que se han venido atenuando las contradicciones entre las ciencias naturales y las sociales, lo cual no implicaba una concepción mecánica de la humanidad, la naturalización del hombre o la positivización de la sociedad, sino más bien la del reconocimiento de su complejidad por las ciencias duras.

[...] las ciencias naturales aparentemente comienzan a acercarse mucho más a lo que había sido despreciado como ciencia social "blanda", más que a lo que se había proclamado como ciencia social "dura". Eso no sólo comienza a modificar el equilibrio de poder en las luchas internas de las ciencias sociales sino que además sirve para reducir la fuerte distinción tanto epistemológica y metodológica, como sobre todo "ontológica" entre ciencias naturales y ciencias sociales como "supercampos" (Hoyos-Vásquez, 2011, p. 6).

En su comprensión de las Ciencias Sociales a partir de la Crítica de la razón pura, de Kant, Guillermo Hoyos-Vásquez planteaba la no contradicción entre los discursos de la ciencia con base en la causalidad y la perspectiva moral del mundo. Lo que propuso, en cambio, fue la importancia de que se adoptaran como perspectivas complementarias que, como

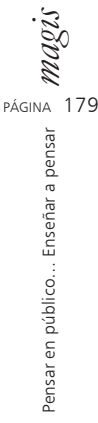

Descripción del artículo | Article description | Description de I'article | Artigo descrição

El artículo ofrece una reseña de algunos de los aportes más significativos de Guillermo Hoyos-Vásquez como gestor y profesor del Doctorado en Ciencias Sociales y Humanas, de la Universidad Javeriana. Describe sus principales reflexiones al interior de los cursos y seminarios en los que participó y su importante impulso tanto al diálogo entre las Ciencias Sociales y las Humanidades como a la noción de interdisciplinariedad en la formación doctoral. 
bellamente lo expresaba, se trataba de la complementariedad de actitudes ante la vida y del desarrollo de saberes también complementarios, como las ciencias naturales, las ciencias sociales y las artes, de modo que se abriesen nuevos horizontes para la reflexión y para todo tipo de discursos, distintos del estrictamente científico de corte positivista.

\section{Los cursos y seminarios como escenarios de formación doctoral}

Lo propio de Guillermo Hoyos-Vásquez como maestro de muchas generaciones fue su genialidad para realizar y compartir una lectura imaginativa de los autores que fundamentaron su pensamiento filosófico - Kant, Husserl y Habermas, entre los principales- y establecer puentes entre la comprensión histórica de los planteamientos para una lectura renovada del presente. En sus clases, la inquietud y preocupación por lo que ocurría en el país y en el mundo fueron la materia viva desde donde invitaba a ver la utilidad de la Filosofía, ya no como un saber abstracto necesitado de una exégesis especializada, sino como una forma de preguntarse y, por medio de la pregunta, despertarse para ir más allá del dogmatismo, la simple opinión o la defensa de visiones que limitasen la autonomía y la capacidad de pensar en la sociedad en que vivimos. Para el profesor Hoyos-Vásquez, la Filosofía es un saber indispensable en un doctorado interdisciplinar que pretende trazar puentes necesarios entre distintos saberes para llegar a perspectivas críticas de la sociedad y de sus prácticas sociales y culturales.

La filosofía, cuanto más inútil, tanto más filosofía es. Pero su utilidad sí consiste en abogar en todo momento por pensar críticamente las diversas situaciones humanas en una perspectiva utópica. Dicho sencillamente, si un filósofo se refiere a la situación colombiana, pone como utopía buscar un cambio en asuntos como la justicia, la igualdad, la paz, la democracia. Pero no es cualquier democracia, no la "seguridad democrática" ni la "prosperidad democrática". Es una democracia sustantiva, más incluyente, una democracia participativa que se tiene que ir construyendo. $Y$ eso lo puede hacer en diálogo con los politólogos, los economistas y los políticos (si es que a los políticos les interesa la filosofía) (Hoyos-Vásquez, 2012).

Como filósofo comprometido con la reflexión pública sobre los graves problemas del país, estaba convencido de que la Filosofía no se debe minusvalorar o silenciar porque, como aclaraba enfáticamente, se elimina uno de los aspectos más importantes del pensamiento crítico y utópico, lo que tiene unos efectos devastadores en el carácter humanista y emancipador de las Ciencias Sociales; de ahí que el diálogo entre estas _Filosofía y Ciencias Sociales - era una tarea urgente para la promoción de sociedades más equitativas, democráticas y humanizadoras.

Sus preguntas incisivas acerca de la formación de doctores en Ciencias Sociales y Humanas cobraron vida en un proyecto de investigación y en tres de los seminarios que coordinó: Paideia para una cultura política, La teoría de la justicia de John Rawls y Jürgen Habermas, la lógica de las ciencias sociales en paradigma comunicativo. A finales de 2012, Guillermo Hoyos-Vásquez insistía en que los estudiantes del doctorado en Ciencias Sociales y Humanas tuvieran la oportunidad de profundizar en el cambio de paradigma propuesto por Jürgen Habermas como un conjunto de principios de renovación de las ciencias sociales. Para ello, quiso ofrecer un seminario junto con Ángela Calvo, discípula y colega suya, con el objetivo de iniciar un estudio riguroso de los principios de la teoría del actuar 
comunicacional y recordar que el propio Jürgen Habermas (1995) había calificado hace mucho tiempo a las ciencias sociales y humanas como "ciencias de la discusión", para distinguirlas de las así llamadas "ciencias positivas"; con ello, buscó enfatizar lo comunicativo de este tipo de saberes (Hoyos-Vásquez, 2009).

\section{Paideia para una cultura política}

Este fue un seminario ofrecido en el segundo semestre de 2011, en el que el profesor Guillermo HoyosVásquez nos advertía sobre cómo la cultura política en Colombia era nuestra asignatura pendiente, que nos enseña que debe haber una actitud pública en la que se opte por una solución política del conflicto y se rechacen de todas formas las soluciones violentas. Consideró entonces que se debería ofrecer un seminario para pensar con seriedad la educación como escenario de la cultura política.

El seminario invitaba a volver a los clásicos (Tagore, Jaeger, Rousseau, Dewey, Freire) para formularnos la siguiente pregunta: ¿cuáles son los fundamentos culturales de la Paideia que revitalizan el sentido de la educación contemporánea, como educación para la constitución de ciudadanía y para el entendimiento entre las culturas? Su interés central tocaba dos puntos neurálgicos del sistema educativo colombiano: la responsabilidad en la formación de ciudadanos y ciudadanos cosmopolitas para los retos que plantean las complejas sociedades contemporáneas y el papel de la educación moral para la ciudadanía en un momento en el que la concepción y la práctica de la educación se encuentran presionadas por criterios meramente económicos:

[...] podríamos preguntar para motivar el seminario, ¿sí creemos al menos nosotros mismos, los educadores colombianos, que vale la pena la educación en valores? ¿No somos nosotros los primeros en dudar si la educación todavía puede servir para civilizar nuestras formas de vida? ¿No será que hemos venido aceptando gradualmente que una educación moral para la ciudadanía es tan inútil que inclusive distrae de los verdaderos objetivos de la educación: invertir en productividad y seguridad, para ser merecedores así de éxito y prosperidad, y eso cuando al menos hay meritocracia? (Hoyos-Vásquez, 2011, p. 1).

Para Guillermo Hoyos-Vásquez, los procesos de formación ciudadana en las escuelas, las universidades y el espacio público eran un tema de relevancia política fundamental sobre el que se debía construir el futuro de un país con problemas tan dramáticos como el nuestro, pero infortunadamente, reconocía que en Colombia se estaba imponiendo una idea diferente de formación ciudadana, basada en una orientación de la educación con fines de lucro que centraba los esfuerzos en la preparación para el trabajo, la productividad y la competitividad. Sostuvo con vehemencia la necesidad de liberar a la educación de la lógica del mercado para pensarla como proceso de formación de ciudadanas y ciudadanos del mundo. Con estas preocupaciones en mente, el profesor Hoyos-Vásquez tenía claro que las Ciencias Sociales y las Humanidades, entre ellas la educación como núcleo impulsor, no podían ser excluidas o disminuidas en su papel protagónico de pensar la nueva sociedad y formar en la sensibilidad moral constitutiva de toda ciudadanía.

\section{El liberalismo político de John Rawls}

En el segundo semestre de 2012, junto con sus colegas Eduardo Pastrana, del Departamento de Relaciones Internacionales y Ángela Calvo, de la Facultad de Filosofía, Guillermo Hoyos-Vásquez ofreció un seminario dedicado a la filosofía política de John Rawls, a quien junto con Jürgen Habermas, se le considera uno de los más influyentes en la discusión sobre filosofía práctica y sobre política desde la década del setenta.

En el seminario se pretendía interpelar los distintos procesos políticos y sociales de la sociedad, en clave de diálogos entre filosofía moral y ciencia política. Para Guillermo Hoyos-Vásquez, el giro decisivo que introdujo Rawls consistió en proponer una base moral -alternativa al utilitarismo dominante- para pensar las condiciones de una sociedad estable y construir una concepción estrictamente política de la justicia: el liberalismo político.

Guillermo Hoyos-Vásquez consideró que el pensamiento liberal de Rawls ofrecía unas bases de hondo contenido pluralista y democrático, por medio de los cuales podría acercar a estudiantes del doctorado a una revisión y un reconocimiento de las actuales sociedades contemporáneas en su amplia diversidad cultural, no solo en términos del accionar comunicativo inspirado en Habermas, sino orientado a debatir cuestiones de alta relevancia para la convivencia en la interculturalidad, tales como el Estado democrático de Derecho, la democracia deliberativa, el papel de la opinión pública y el consenso entrecruzado entre diversas doctrinas comprensivas de vida buena, a partir del hecho de que en las sociedades contemporáneas interactúan varias concepciones englobantes del sentido de la vida, tanto religiosas como morales y filosóficas.

Según el profesor Hoyos, el pluralismo razonable al que alude Rawls es lo que facilitaría dicho consenso en torno a los principios de justicia en una concepción política, en particular, basada en la igualdad de libertades y de oportunidades y la distribución equitativa de bienes primarios. Para el caso colombiano y otros países con alto grado de inequidad, la concepción política 
de justicia y sociedad civil ayudan a comprender los motivos de desorden social y de violencia, asociados, en gran parte de los casos, con la no realización concreta de los derechos fundamentales (Hoyos-Vásquez \& Vargas Guillén, 1997) y a las situaciones de injusticia que prevalecen y carcomen la esperanza de mucha gente.

\section{Universidad colombiana y sociedad del conocimiento: un estudio comprehensivo sobre la idea de universidad}

Guillermo Hoyos-Vásquez hizo un aporte significativo al pensamiento sobre la idea de universidad y en particular sobre su ethos en el contexto de la modernidad. Su trabajo intenso con distintas instituciones de la educación superior colombiana, así como en comisiones y foros, le proveyó de un escenario propicio para contribuir con una visión incisiva y a la vez propositiva de la universidad colombiana, en el que advirtió sobre la crisis del modelo único de universidad obsesionada por la modernización, los indicadores y la tecnología:

Las expectativas con respecto a la evolución de la educación superior parecen responder a un modelo único de universidad, fruto de la modernidad ilustra$\mathrm{da}$, la que encarna aquella idea de universidad que quisiéramos sobreviviera a todas las crisis; la que nos promete la ciencia y la tecnología, clave para acceder a la apertura y a la globalización. ¿Y por qué una universidad moderna no puede darnos en el campo de la educación moral, científica, cultural y política la clave para solucionar todos los problemas relacionados con la sociedad civil? Así que muchos pensaron que sólo faltaba con modernizar nuestras universidades según el modelo de universidad liberal.

Pero es precisamente el modelo único de universidad el que está en crisis, y no propiamente las universidades por no adecuarse todas a dicho modelo. Es el programa de su modernización sin más, sin tener en cuenta otras alternativas, ni los alcances de la modernidad, ni sus mismos límites, lo que ha provocado esta crisis (Hoyos-Vásquez, 1998, p. 18).

En 2010, motivó al grupo de profesores del Eje 3: Sociedad de la información, comunicación y educación ${ }^{2}$ a emprender un estudio comprehensivo sobre la idea de universidad, que tensionara las relaciones entre esta y la sociedad. Para ello, nos introdujo en la lectura del libro de M. C. Nussbaum (2010), Sin fines

2 Los profesores que conforman el Eje 3 son: Luis Ignacio Sierra, Iliana Hernández, Carlos Gaitán, Fabiola Cabra, Jaime Alejandro Rodríguez, María Caridad García, Rafael Campo, Gloria Marciales, Eduardo Rueda, Martha Lozano y Guillermo Hoyos-Vásquez, vinculado desde sus inicios. de lucro: por qué la democracia necesita de las humanidades; a partir de él, nos adentramos en los argumentos sobre la crisis de la educación centrada en los modelos de rentabilidad económica o educación con ánimo de lucro y el papel de las humanidades en las universidades y su aporte a la conformación de sociedades democráticas.

La afirmación explícita de que la democracia necesita de las humanidades ayuda a caracterizar la ciudadanía en términos de convivencia, cooperación, calidad de vida, a la vez condiciones y frutos de una auténtica democracia como forma de vida moderna. Ello, naturalmente, no excluye la educación profesional pero sí pretende ser para ella un punto de partida, de suerte que los criterios que primen en el mundo profesional no sean solo los de la mayor ganancia, sino los que conformen el sentido de sociedad bien ordenada. Una educación humanista desarrollada en los tres pasos (persona, sociedad y cultura) cualifica la democracia y la abre al cosmopolitismo intercultural (Hoyos-Vásquez, 2011, p. 6).

El primer tomo de la investigación adelantada fue publicado en 2013 bajo el título de Universidad y sociedad: aproximaciones críticas, tensiones y desafíos, en el que se recogen los aportes de profesores colombianos y latinoamericanos acerca de las tendencias recientes en el desarrollo de la universidad.

De este intenso y desafiante trabajo de análisis de la universidad contemporánea hay dos reflexiones finales que Guillermo Hoyos-Vásquez quiso dejarnos como programa de investigación: por un lado, no abandonar un trabajo docente e investigativo donde prevalezca una siempre renovada relación de complementariedad entre filosofía y educación, para que no se reduzca a indicadores de calidad y competencias y, por otro, apostar a la construcción de un ethos universitario que esté a la altura de los desafíos éticos y culturales que están viviendo Colombia y el mundo.

\section{La presencia viva de un amigo y colega entrañable}

La noticia de la muerte de Guillermo HoyosVásquez me tomó por sorpresa. Apenas un mes antes estábamos muy interesados en su próximo seminario para el primer semestre de 2013. Nunca quise pensar en su ausencia, siempre recordaba su amable sonrisa, su espléndido sentido del humor y sincero interés en los "muchachos", como se refería a sus estudiantes.

Su voz como mensaje de denuncia no se apagará y tampoco el legado con el que despertó en muchos de nosotros el imperativo de resistir a todo proceso de deshumanización, de tener la capacidad de resolver 
los conflictos mediante la comunicación con el adversario y la posibilidad de perdonar como virtud cívica para lograr la paz, ya que, como lo expresa en uno de los últimos artículos publicados en la prensa nacional en 2012, "Es el perdón mismo el que cura". Continúa Guillermo Hoyos-Vásquez:

Si se considera la situación creada por el 11 de septiembre del 2001, es evidente que sin la cultura del perdón como virtud cívica se seguirán atizando en el ámbito mundial nuevas violencias, nuevos terrorismos, nuevas guerras. Y si pensamos en Colombia, es claro que dos gobiernos de seguridad democrática en nada favorecieron la virtud cívica del perdón.

Creo que en este momento lo más importante es que los colombianos nos preguntemos qué tan alta tenemos la virtud cívica de la cultura del perdón. Si llegamos a la actitud de querer poder perdonar lo imperdonable o si pensamos que el tema de la impunidad es asunto de justicia, no como equidad, sino como castigo, traducida en años de cárcel (ArangoRestrepo, 2012).

Con la referencia a Derrida — para quien el perdón es de lo imperdonable-, el profesor Hoyos-Vásquez invita a reflexionar sobre la naturaleza y el significado que tiene el perdón como virtud cívica para la solución del conflicto y para pensarnos en un escenario postconflicto en Colombia. Es un tema polémico que se confunde con impunidad y tiende a la frivolización en los sistemas burocráticos de la justicia y la política; no obstante, para el profesor Hoyos-Vásquez se presenta como tema urgente y necesario, en particular, porque hay quienes pretenden alcanzar la paz por la guerra. A ellos les recuerda, en alusión a las palabras de Kant, que "lo grave de la guerra radica es que crea más personas malas que las que elimina" (Kant, 1966, p. 82).

Al profesor Hoyos-Vásquez lo recordaremos siempre como un maestro, intelectual íntegro y amigo comprometido, que conquistó nuestro cariño y admiración con su honestidad e inteligencia. También lo recordaremos por el privilegio de haber compartido tantos momentos y lecciones de vida, de tan profundo sentido ético y político, para nuestro país. En el Doctorado en Ciencias Sociales y Humanas y sus colegas del Eje 3 le estaremos eternamente agradecidos por su testimonio magistral de dedicación, sabiduría y amistad solidarias. iLe recordaremos por siempre con afecto!

\section{Sobre la autora}

Fabiola Cabra-Torres es licenciada en Español-Inglés, magíster en Educación y doctora en Innovación Educativa. Es profesora asociada de la Facultad de Educación y del Doctorado en Ciencias Sociales de la Pontificia Universidad Javeriana, de Bogotá. Investigadora externa del Equipo Innova, de la Universidad de Deusto (España). Líneas de investigación: evaluación en la educación superior, competencias informacionales y aprendizaje en la sociedad de la información.

\section{Referencias}

Arango Restrepo, C. (2012). Cultura política, nuestra asignatura pendiente: Guillermo Hoyos-Vásquez. [Entrevista con Guillermo Hoyos-Vásquez, filósofo]. El Tiempo. Recuperado de http://m.eltiempo.com/politica/ la-poltica-desde-la-filosofa-por-guillermo-hoyos/11911681

Habermas, J. (1995). Sobre la situación de las ciencias sociales y ciencias humanas (Geisteswissenschaften) en Alemania. Una mirada a su evolución en la postguerra. Diálogo Científico, 4 (1/2), 11-21. 
Hoyos-Vásquez, G. \& Vargas Guillén, G. (1996). La teoría de la acción comunicativa como nuevo paradigma de investigación en ciencias sociales: las ciencias de la discusión. Bogotá: Icfes.

Hoyos-Vásquez, G. (1998). El ethos de la universidad. UIS-Humanidades, $27(1), 13-23$.

Hoyos-Vásquez, G. (2008). La responsabilidad del pensar. Homenaje a Guillermo Hoyos-Vásquez. Ideas y valores, 56 (136), 145-147.

Hoyos-Vásquez, G. (2009). Educación para un nuevo humanismo. magis, Revista Internacional de Investigación en Educación, 1 (2), 425-433.

Hoyos-Vásquez, G. (enero-abril, 2011). Educación y ética para una ciudadanía cosmopolita. Revista Iberoamericana de Educación, 55, 1-10.

Hoyos-Vásquez, G. (septiembre, 2011). Manifiesto por las ciencias sociales y las humanidades. En XI Congreso de Investigación llevado a cabo por la Vicerrectoría Académica de la Pontificia Universidad Javeriana, Bogotá.

Hoyos-Vásquez, G. (2011). Seminario Paideia. Para una cátedra política. Plan de estudios. Pontificia Universidad Javeriana, Bogotá, Colombia.

Hoyos-Vásquez, G. (2012). Sociedad, política y bioética: ¿cómo debe ser la filosofía para salvar la bioética en Latinoamérica? En F. J. León (coord.), Bioética y sociedad en Latinoamérica. Santiago de Chile: Fundación Interamericana Ciencia y Vida.

Hoyos-Vásquez, G. (2012, octubre 22). El perdón es de lo imperdonable. El Tiempo. Recuperado de http://www.eltiempo.com/justicia/ARTICULO-WEB-NEW_NOTA_INTERIOR-12327159.html

Kant, I. (1966). La paz perpetua. Madrid: Aguilar.

Nussbaum, M. (1997). Cultivating Humanity. A Classical Defense of Reform in Liberal Education. Cambridge: Harvard University Press.

Nussbaum, M. (2010). Sin fines de lucro: por qué la democracia necesita de las humanidades. Madrid: Paidós.

Marciales Vivas, G. (coord.) (2008). Documento curricular del Doctorado en Ciencias Sociales y Humanas, Pontificia Universidad Javeriana, Bogotá. 